\title{
Measurement of the photoelectron-collection efficiency in noble gases and methane
}

\author{
L.C.C. Coelho ${ }^{a}$, H.M.N.B.L. Ferreira ${ }^{a}$, J.A.M. Lopes ${ }^{a}$,b,*, T.H.V.T. Dias ${ }^{a}$, L.F.R. Ferreira ${ }^{a}$, \\ J.M.F. dos Santos ${ }^{\mathrm{a}}$, A. Breskin ${ }^{\mathrm{c}}$, R. Chechik ${ }^{\mathrm{c}}$ \\ ${ }^{a}$ Departamento de Física, Faculdade de Ciencias e Technologia, Universidade de Coimbra, 3004-516 Coimbra, Portugal \\ ${ }^{\mathrm{b}}$ Instituto Superior de Engenharia de Coimbra, 3030-199 Coimbra, Portugal \\ ${ }^{\mathrm{c}}$ Weizmann Institute of Science, 761000 Rehovot, Israel
}

Available online 29 July 2007

\begin{abstract}
The photoelectron-collection efficiency from photocathodes in noble gases and methane is experimentally investigated. The ratio between the number of transmitted photoelectrons in the gas media and in vacuum is determined as a function of the applied reduced electric field $E / p$, where $p$ is the gas pressure. Results are presented for $\mathrm{He}, \mathrm{Ne}, \mathrm{Ar}, \mathrm{Xe}, \mathrm{Kr}$ and $\mathrm{CH}_{4}$.
\end{abstract}

(C) 2007 Elsevier B.V. All rights reserved.

PACS: 51.10. +y; 29.40.Mc; 79.60.Bm

Keywords: Photoemission; Noble gases; Methane; Electron backscattering

\section{Introduction}

Radiation detectors are often based on the production of scintillation, and a photosensor device is used where photoelectrons are extracted from a photocathode and collected at an anode to produce a pulse. Applications of Cherenkov detectors, gas scintillation counters, gas photomultipliers and other VUV photosensors have been implemented. In most cases, the photocathodes are in contact with a gaseous atmosphere, allowing for backscattering of photoelectrons to the photocathode and reducing the effective quantum efficiency.

Several studies have been performed, either by simulation or experimentally, to determine the photoelectroncollection efficiency in different gases, when compared to vacuum [1-9]. The photoelectron-collection efficiency depends on the gas, the electric field intensity at the photocathode surface and the photon energy incident on the photocathode.

\footnotetext{
*Corresponding author. Departamento de Física, Faculdade de Ciencias e Technologia, Universidade de Coimbra, 3004-516 Coimbra, Portugal. Tel.: + 351239410600 ; fax: + 351239829158 .

E-mail address: fcjam@gian.fis.uc.pt (J.A.M. Lopes).
}

In the case of noble gas filled photosensors, a particularly significant reduction in the collected charge is observed due to the backscattering of the photoelectrons by the gas atoms [2-5,7-9]. Pure noble gases are important for its large scintillation, easy handling and purification and the use of solid photocathodes in pure rare gas is being implemented [5,10-12]. Up to now, the photoelectroncollection efficiency has been studied experimentally only for pure $\operatorname{Ar}[2,3,5]$, while simulation studies have been performed for $\operatorname{Ar}[2,4,7,8]$ and for Ne and Xe [7,8].

In the present work, the backscattering effects in pure $\mathrm{He}, \mathrm{Ne}, \mathrm{Ar}, \mathrm{Kr}, \mathrm{Xe}$, and $\mathrm{CH}_{4}$ are investigated. For that purpose, a $500 \mathrm{~nm}$ thick reflective CsI photocathode is used. The photoelectron emission is induced by a Hg lamp (185 nm centered, $5 \mathrm{~nm}$ FWHM). The photoelectroncollection efficiency is determined as a function of the applied reduced electric field $E / p$, where $p$ is the gas pressure.

\section{Experimental set up and procedure}

The experimental set up used for this work is depicted in Fig. 1. A small vacuum chamber was assembled with the 


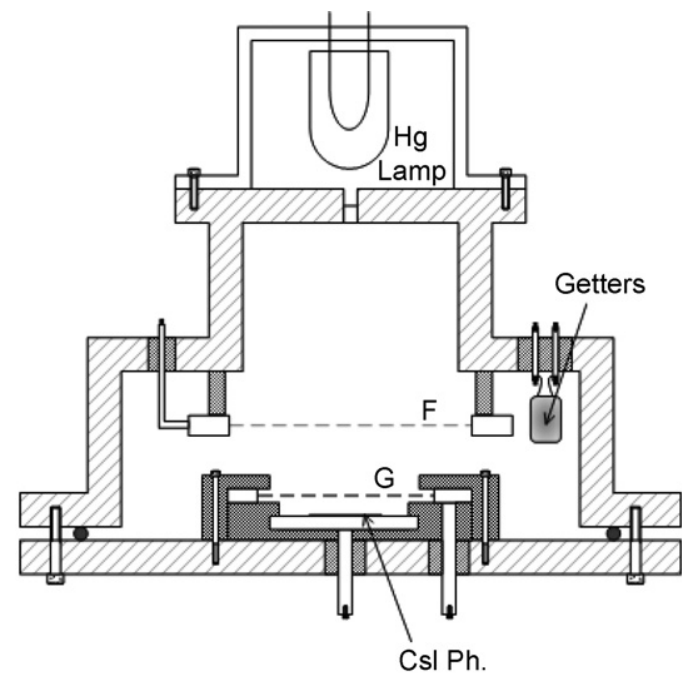

Fig. 1. Experimental set-up for the measurement of the photoelectroncollection efficiency in a gaseous atmosphere.

CsI photocathode deposited on a gold plated stainless steel plate, placed at a distance of $4 \mathrm{~mm}$ from the photoelectroncollecting grid $\mathrm{G}$. A second grid $\mathrm{F}$ is placed above grid $\mathrm{G}$ so that an electric field can be established between the two grids to guarantee full photoelectron collection when operating under vacuum. The chamber is made of stainless steel except for the Macor ceramic used for electrical insulation of the stainless steel plate, the grids and the feedthroughs. The grids are made of stainless steel wire with $80 \mu \mathrm{m}$ diameter and $900 \mu \mathrm{m}$ pitch.

The chamber is filled with the gas under test, at atmospheric pressure, and is sealed during the measurements. Small getter units with a built-in resistor (SAES ST172/HI/7) are used to keep a high level of gas purity. The CsI photocathode is irradiated with the VUV photons and the photocurrent is measured in the photocathode as a function of the grid $G$ voltage, using a Keithley Instruments $610 \mathrm{C}$ electrometer. The photocathode plate was operated at ground potential. To check for any possible photocathode degradation during measurements, the photocurrent under vacuum is measured before and after the measurements in gaseous atmosphere.

For each gas, the photoelectron-collection efficiency was measured as the ratio between the photocurrent values obtained while operating the photocathode in the gas and vacuum for the same applied field. For each gas, measurements were performed in different gas fillings in order to obtain an average value for the photoelectroncollection efficiency and the corresponding error.

\section{Experimental results and discussion}

Experimental results for the photoelectron-collection efficiency in $\mathrm{Ar}$ and $\mathrm{CH}_{4}$ as a function of the electric field above the photocathode surface are shown in Fig. 2, together with other results presented in the literature.

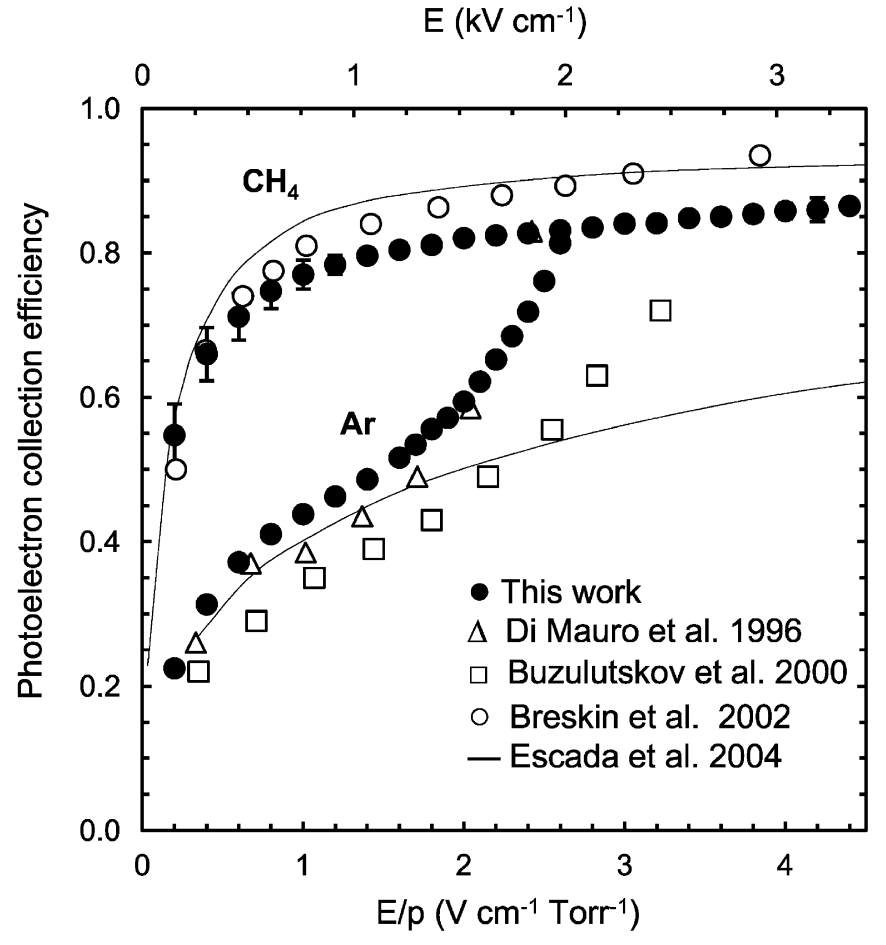

Fig. 2. Photoelectron-collection efficiency in $\mathrm{Ar}$ and $\mathrm{CH}_{4}$ as function of the electric field applied in the gas when the CsI phocathode is irradiated with a $\mathrm{Hg}(\mathrm{Ar})$ lamp. The symbols correspond to experimental values while the solid lines represent Monte Carlo simulation results.

The collecting effect of the field is observed for both gases. The results obtained in this work are in agreement with those presented in the literature. The more noticeable differences between the experimental values for argon could be attributed to different levels of gas impurities. For Ar, the onset of gas excitation $\left(E / p>1 \mathrm{~V} \mathrm{~cm}^{-1} \mathrm{Torr}^{-1}\right)$ and photon feedback effects prevent the observation of the slow increase in photoelectron collection with increasing field as observed for methane. Instead, a fast increase of the photoelectron collection with the electric field is observed above that threshold.

In Fig. 3 we present the experimental results obtained for the electron-collection efficiencies in $\mathrm{He}, \mathrm{Ne}, \mathrm{Ar}, \mathrm{Kr}$ and $\mathrm{Xe}$ as a function of the electric field above the CsI photocathode. Except for $\mathrm{He}$, all the other gases present a fast increase in the photon-collection efficiency due to photon feedback, for reduced electric fields above the gas excitation threshold: $\sim 0.5 \mathrm{~V} \mathrm{~cm}^{-1} \mathrm{Torr}^{-1}$ for $\mathrm{Ne}, \sim 1 \mathrm{~V} \mathrm{~cm}^{-1} \mathrm{Torr}^{-1}$ for $\mathrm{Ar}, \mathrm{Kr}$ and $\mathrm{Xe}$ and $\sim 2 \mathrm{~V} \mathrm{~cm}^{-1} \mathrm{Torr}^{-1}$ for He [13]. The broken lines represent a logarithmic function fitted to the experimental data below the gas scintillation threshold. This logarithmic trend is observed in Monte Carlo simulation results of photoelectron-collection efficiencies calculated for any gaseous atmosphere [7-9]. Assuming that this trend presents a typical behavior for the photoelectron-collection efficiency dependence on the electric field above the photocathode surface, the dashed curves of Fig. 3 present 


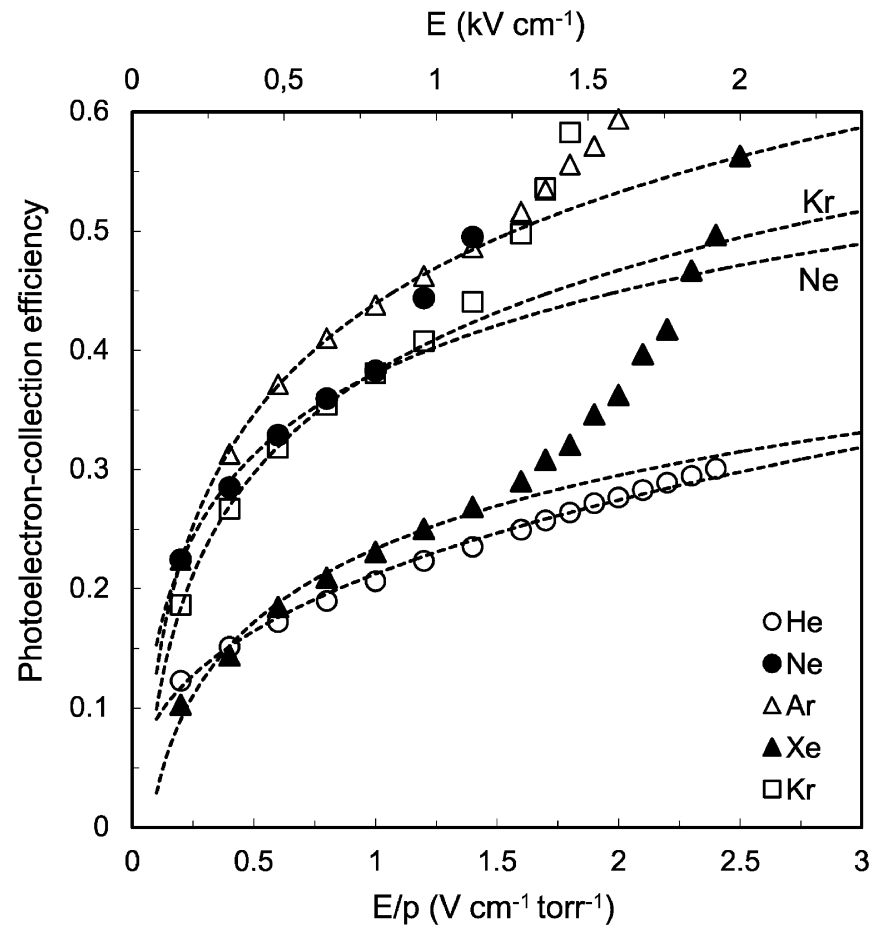

Fig. 3. Photoelectron-collection efficiencies in $\mathrm{He}, \mathrm{Ne}, \mathrm{Ar}, \mathrm{Xe}$ and $\mathrm{Kr}$ as a function of the pressure reduced applied electric field.

a good estimation for the real photoelectron-collection efficiency in noble gases without the presence of photon feedback, even at high electric fields. This situation can occur whenever the path travelled by photoelectrons in the region above the photocathode is of the order of few tens of $\mu \mathrm{m}$ so that photoelectrons produce only a few scintillation photons in that path, e.g. as in micro patternbased photosensors operating with reflective photocathodes. As an example, about five photons are produced at $4 \mathrm{~V} \mathrm{~cm}^{-1} \mathrm{Torr}^{-1}$ for paths $\sim 100 \mu \mathrm{m}$ in Xe at atmospheric pressure.

Fig. 3 shows that the photoelectron-collection efficiency for $\mathrm{He}$ and $\mathrm{Xe}$ only reaches values about $30 \%$ at reduced electric fields of $4 \mathrm{~V} \mathrm{~cm}^{-1} \mathrm{Torr}^{-1}$, a factor of two lower than that obtained for $\mathrm{Ar}, \mathrm{Kr}$ or $\mathrm{Ne}$, and about a factor of three lower than that for the molecular gas $\mathrm{CH}_{4}$. This fact has to be taken into account in the operation of solid photocathodes in xenon (e.g. in applications to Xe dual-phase detectors for dark matter search), since the effective quantum efficiency of a CsI photocathode operated in a xenon atmosphere will be well below $10 \%$ for the distribution of the $\mathrm{Hg}$ lamp, centered at $185 \mathrm{~nm}$. Fig. 3 also shows that the photoelectron-collection efficiency in $\mathrm{Ne}$ is lower than that for Ar. If the same relative effect is observed for xenon scintillation light, centered at $172 \mathrm{~nm}$, then this could be one of the reasons for the poorer performance obtained with a microstrip-based photosensor operating in $\mathrm{Ne}-\mathrm{Xe}$ atmospheres [10] when comparing with those obtained in $\mathrm{Ar}-\mathrm{Xe}$ mixtures [14].

\section{Conclusions}

We have measured the photoelectron-collection efficiency of a CsI photocathode operating in noble gas atmospheres and methane, when irradiated with a $\mathrm{Hg}$ lamp. Our results confirm the low photoelectron-collection efficiencies that are achieved in noble gases. He and $\mathrm{Xe}$ present the worst cases with the photoelectron-collection efficiency achieving values around $30 \%$ for reduced electric fields about $4 \mathrm{~V} \mathrm{~cm}^{-1} \mathrm{Torr}^{-1}$; the values for $\mathrm{Ne}$ and $\mathrm{Kr}$ are around $50 \%$, while for Ar, the most favorable case, the achieved values are about $60 \%$. This is a drawback for the operation of solid photocathodes in noble gas atmosphere, when comparing with the values around $90 \%$ that are achieved in molecular gases such as $\mathrm{CH}_{4}$. The effective quantum efficiency of a CsI photocathode operating in xenon atmosphere is well below $10 \%$ for this spectral distribution.

For reduced electric fields above the gas excitation threshold, secondary scintillation photons are produced in the gas by photoelectrons in their path above the photocathode, and photon feedback effects will occur. However, if this path length is short enough, so that the number of secondary photons that are produced is small, these effects will become negligible.

\section{Acknowledgments}

This work was supported by FEDER and POCI2010 programs, through FCT (Fundação para a Ciência e Tecnologia), project POCI/FP/63418/2005, by the MINERVA Foundation and by the Israel Science Foundation project $151 / 01$. A. Breskin is the W.P. Reuther Professor of Research in peaceful use of atomic energy.

\section{References}

[1] A. Breskin, A. Buzulutskov, R. Chechik, A. Di Mauro, E. Nappi, G. Paic, F. Piuz, Nucl. Instr. and Meth. A 367 (1995) 342.

[2] A. Di Mauro, E. Nappi, F. Posa, A. Breskin, A. Buzulutskov, R. Chechik, S.F. Biagi, G. Paic, F. Piuz, Nucl. Instr. and Meth. A 371 (1996) 137.

[3] A. Buzulutskov, L. Shekhtman, A. Bressan, A. Di Mauro, L. Ropelewski, F. Sauli, S. Biagi, Nucl. Instr. and Meth. A 433 (1999) 471

[4] A.V. Phelps, Z.L. Petrovic, Plasma Sources Sci. Technol. 8 (1999) 21.

[5] A. Buzulutskov, A. Breskin, R. Chechik, G. Garty, F. Sauli, L. Shekhtman, Nucl. Instr. and Meth. A 443 (2000) 164.

[6] A. Breskin, A. Buzulutskov, R. Chechik, Nucl. Instr. and Meth. A 483 (2002) 670.

[7] P.J.B.M. Rachinhas, J.A.M. Lopes, T.H.V.T. Dias, F.P. Santos, A.D. Stauffer, C.A.N. Conde, in: Proceedings of MC2000 International Conference on Advanced Monte Carlo for Radiation Physics, Particle Simulation and Applications, Springer, Heidelberg, 2001, p. 535

[8] T.H.V.T. Dias, P.J.B.M. Rachinhas, J.A.M. Lopes, F.P. Santos, L.M.N. Távora, C.A.N. Conde, A.D. Stauffer, J. Phys. D 37 (2004) 540.

[9] J.M.D. Escada, P.J.B.M. Rachinhas, T.H.V.T. Dias, J.A.M. Lopes, F.P. Santos, C.A.N. Conde, A.D. Stauffer, in: J.A. Seibert (Ed.), 2004 IEEE Nuclear Science Symposium \& Medical Imaging 
Conference, 16-22 October, Roma, Italy. Conference Record, vol. 1, 2004, pp. 559-563.

[10] D.S.A.P. Freitas, J.F.C.A. Veloso, C.M.B. Monteiro, J.M.F. dos Santos, C.A.N. Conde, Nucl. Instr. and Meth. A 505 (2003) 228 (and references therein).

[11] L. Periale, V. Peskov, P. Carlson, C. Iacobeause, T. Francke, N. Pavlopoulos, F. Pietropaolo, T. Sokolova, P. Picchi, Nucl. Instr. and Meth. A 497 (2003) 242.
[12] T. Meinschad, L. Ropelewski, F. Sauli, Nucl. Instr. and Meth. A 547 (2005) 342.

[13] M.A. Feio, A.J.P.L. Policarpo, M.A.F. Alves, Jpn. J. Appl. Phys. 8 (1982) 1184.

[14] L.M.P. Fernandes, D.S.A.P. Freitas, A.M.F. Trindade, J.F.C.A. Veloso, C.M.B. Monteiro, L.F. Requicha Ferreira, J.M.F. dos Santos, IEEE Trans. Nucl. Sci. NS-51 (2004) 1509. 